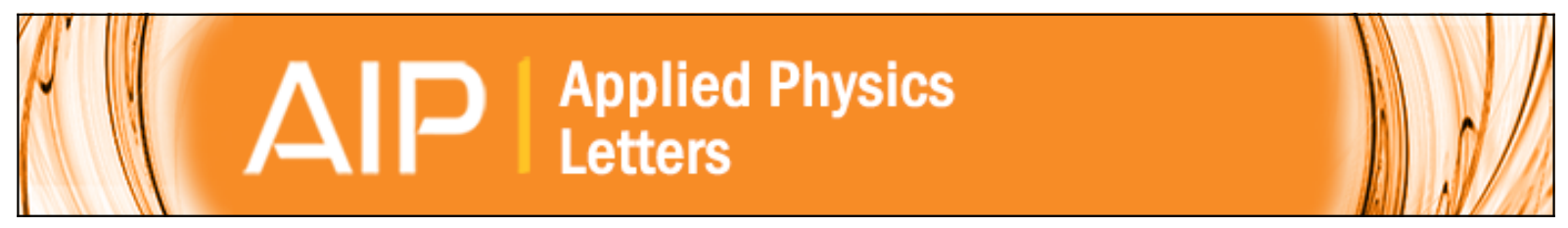

\title{
Fabrication of small laterally patterned multiple quantum wells
}

A. Scherer and H. G. Craighead

Citation: Applied Physics Letters 49, 1284 (1986); doi: 10.1063/1.97387

View online: http://dx.doi.org/10.1063/1.97387

View Table of Contents: http://scitation.aip.org/content/aip/journal/apl/49/19?ver=pdfcov

Published by the AIP Publishing

\section{Articles you may be interested in}

Phosphor-free white light-emitting diode with laterally distributed multiple quantum wells

Appl. Phys. Lett. 92, 091110 (2008); 10.1063/1.2890492

Lateral confinement in $\mathrm{ZnSe} / \mathrm{ZnCdSe}$ quantum wells grown on patterned substrates

Appl. Phys. Lett. 72, 575 (1998); 10.1063/1.120763

Negative differential conductance in lateral double-barrier transistors fabricated in strained Si quantum wells Appl. Phys. Lett. 70, 2422 (1997); 10.1063/1.118891

Lateral quantum well wires fabricated by selective metalorganic chemical vapor deposition Appl. Phys. Lett. 57, 1209 (1990); 10.1063/1.103487

Lateral refractive index step in GaAs/AIGaAs multiple quantum well waveguides fabricated by impurity-induced disordering

Appl. Phys. Lett. 55, 1412 (1989); 10.1063/1.101610

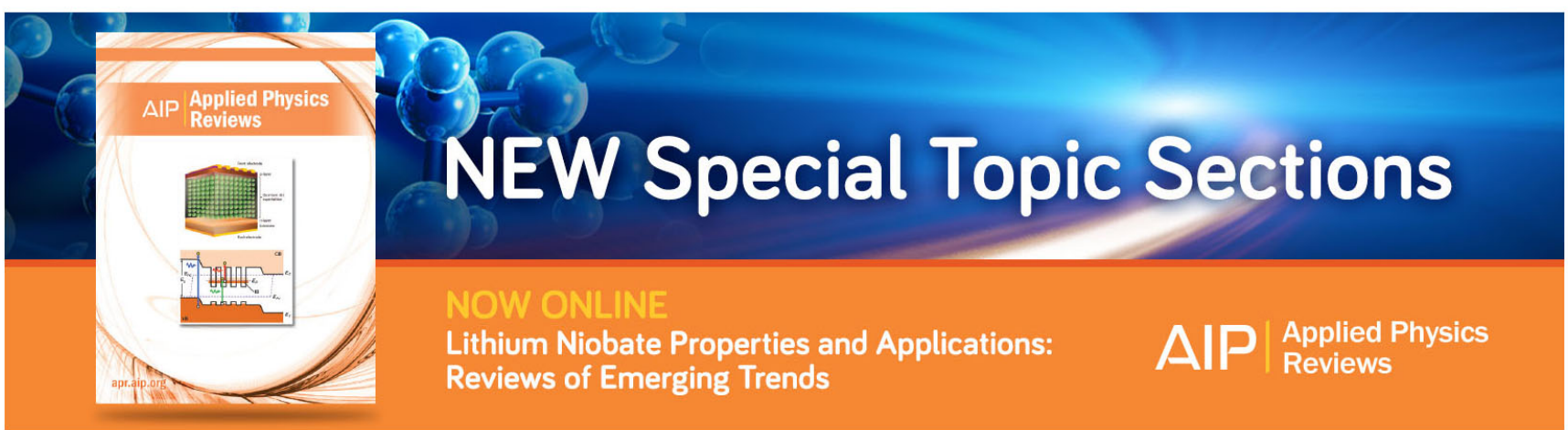




\title{
Fabrication of small laterally pattemed multiple quanchm wells
}

\author{
A. Scherer and H. G. Craighead \\ Bell Communication Research, Red Bank, New Jersey 07701
}

(Received 17 July 1986; accepted for publication 15 September 1986)

\begin{abstract}
A technique of high voltage electron beam lithography and $\mathrm{BCl}_{3} / \mathrm{Ar}$ reactive ion etching for laterally patterning $\mathrm{GaAs} / \mathrm{Al}_{0,3} \mathrm{Ga}_{0.7}$ As multiple quantum wells is described. The resulting structures were analyzed using scanning electron microscopy and a novel reflection electron microscopy technique, and their geometries are shown. Narrow columns $40 \mathrm{~nm}$ in diameter etched $230 \mathrm{~nm}$ through the quantum wells were reproducibly fabricated.
\end{abstract}

Microstructural control of GaAs and lattice matched AlGaAs by epitaxial growth techniques has enabled tailoring the electronic properties of these semiconductor materials. This has led to the design of new electronic and optoelectronic devices such as high electron mobility transistors and heterostructure lasers, and has revealed new physical phenomena such as the quantized Hall effect. Advances in technology for lateral patterning have motivated new research directions and device concepts to take advantage of geometrical confinement in more than one dimension. ${ }^{1,2}$ The degree of structural control obtainable in lateral patterning has not reached the nanometer dimensions attained by controlled growth, but high-resolution lateral patterning is improving and the range of geometries and materials increasing. ${ }^{3.4}$

In this letter, we describe the fabrication, analytical techniques, and exemplary structures of the smallest $(<50$ $\mathrm{nm}$ ) compound semiconductor heterostructures. To accomplish patterning on such a fine scale, a variety of problems such as mask erosion, undercutting, resolution, and etch rate control had to be overcome. Some of the techniques used for the first time in III-V semiconductors include electron beam lithography at energies greater than $150 \mathrm{keV}$, fluoride etch masks and resists, and high-resolution reflection electron imaging of structures. Examples of small structures include arrays of <40-nm-diam "quantum disks" etched in $\mathrm{GaAs} / \mathrm{AlGaAs}$ multiple quantum well material. Since the lateral dimension of these structures is close to the thickness of the quantum wells, photoluminescence spectra can be observed, which are very different from the grown layered material. ${ }^{5}$

We have established processes specific to the fabrication of ultrasmall, high-quality compound semiconductor samples. Electron beam lithography was used to define the small lateral dimensions. ${ }^{6}$ Electron beam voltages in excess of 150 $\mathrm{keV}$ were used to avoid broadening of individual disks through exposure to backscattered electrons and to minimize proximity effects or exposure variation over the sample. A scanning transmission electron microscope (STEM) was used to pattern the resist to attain beam sizes from 2 to 6 $\mathrm{nm}$. Beam currents of $2-10 \mathrm{pA}$ were obtained with a $\mathrm{LaB}_{6}$ electron source, and the sample was focused in the previously aligned STEM by bringing the sample into the eucentric height, imaging a gold grid pattern under the polymethylmethacrylate (PMMA) resist with the backscattered electron detector.

Although negative resists can be used to directly expose and, subsequently, etch the substrate, the most reproducible technique was a lift-off procedure using 70-nm-thick PMMA as a positive resist material. This resist was exposed by doses of $0.20 \mathrm{pC} / \mu \mathrm{m}$. The resist was developed by a $10-\mathrm{s}$ immersion in a 3:7 cellusolve:methanol mixture followed by a water rinse. A liftoff (Fig. 1) was used to provide the etch mask on the sample. To minimize the thickness of the etch mask and thereby gain more resolution in this lift-off procedure, either a $15-\mathrm{nm}$ nickel or a $10-\mathrm{nm} \mathrm{SrF}_{2}$ mask was used.

To structure the multiple quantum well material, reactive ion etching (RIE) was used. Since AlGaAs etches differently from GaAs, etching conditions which limit the lateral erosion of GaAs have to be chosen. This was accomplished in a 9:1 $\mathrm{Ar}: \mathrm{BCl}_{3}$ gas mixture at pressures of 15 mTorr at $50 \mathrm{~W}$ for a 6 -in. substrate in a standard planar RIE system. These etching conditions were found to provide similar etch rates for AlGaAs and GaAs, and did not substantially undercut the GaAs. Etch rates of approximately 30 $\mathrm{nm} / \mathrm{min}$ were obtained with these conditions.?

Scanning electron microscopy (SEM) and reflection electron microscopy (REM) were performed in a STEM

\section{Liftole Procedure}

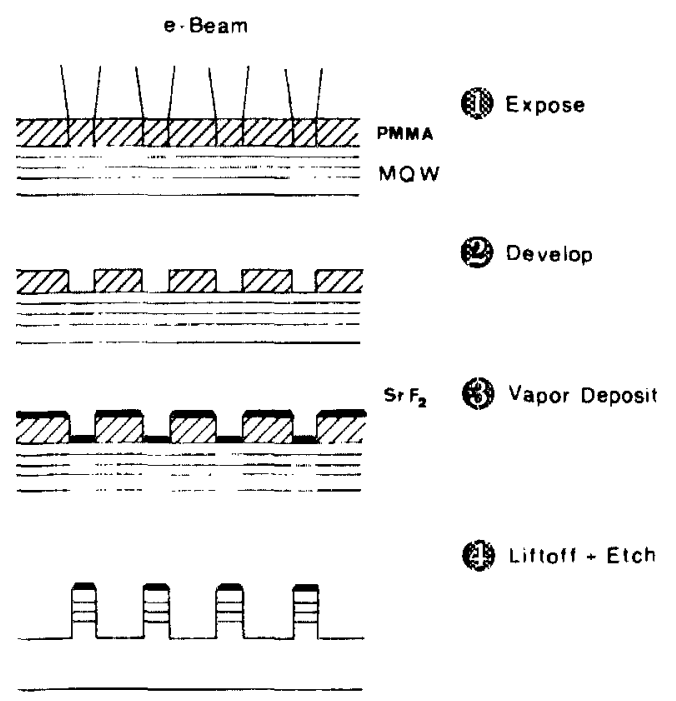

FIG. 1. Schematic of lift-off process used with PMMA resist. 


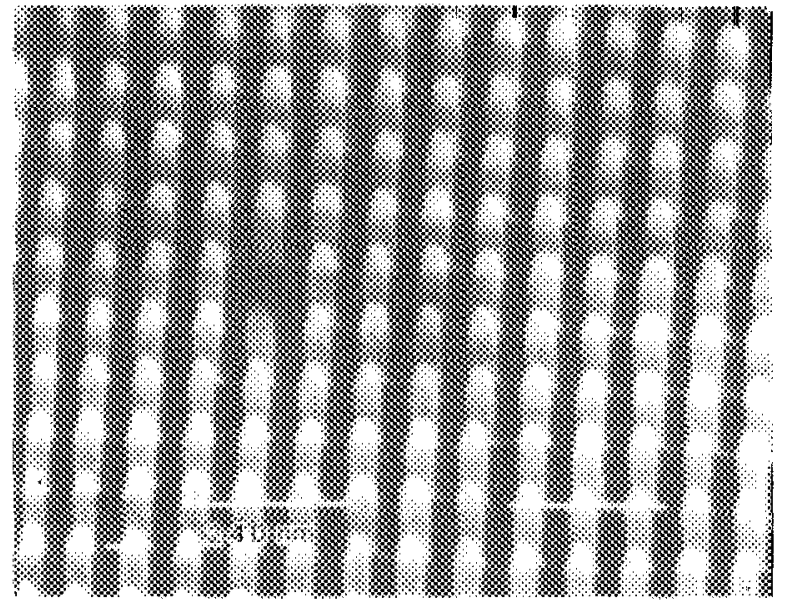

(a)

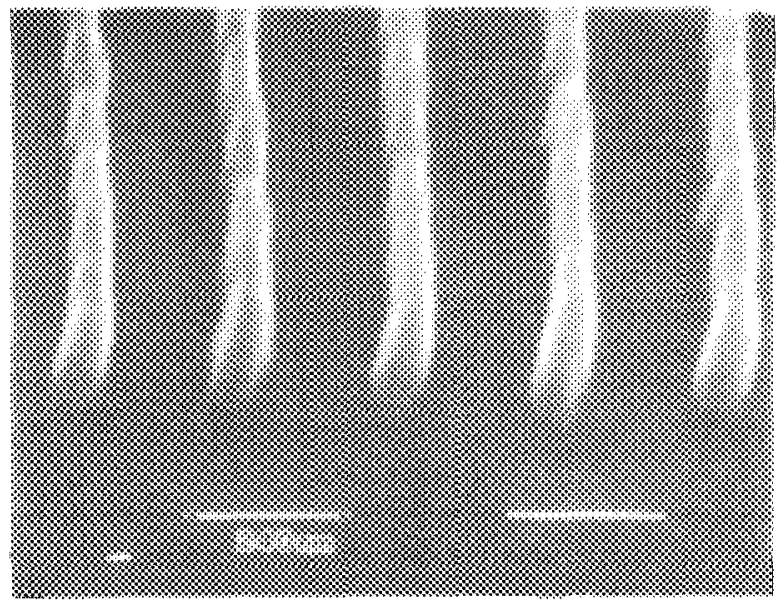

(b)

FIG. 2. (a) SEM micrograph of columns etched into GaAs/AlGaAs multiple quantum well material, using $\mathrm{SrF}_{2}$ as an etch mask. (b) SEM micrograph of lines etched into the same material (micrograph taken at $45^{\circ}$ ).

and were used to measure the dimensions of the patterned structures. The quality of the etched substrate was investigated with reflection electron diffraction both of patterned and of unpatterned areas. Photoluminescence spectra taken from overexposed and patterned areas and compared with the spectra of the original material (to describe the quality of the quantum wells after electron-beam irradiation) show an apparent increase in the luminescence efficiency of the patterned material..$^{5}$

A sample consisting of several 15-, 6-, and 3-nm-thick quantum wells was patterned using $\mathrm{SrF}_{2}$ as an etch mask. This sample was then etched $200 \mathrm{~nm}$ by RIE producing an array of columns with diameters of approximately $40 \mathrm{~nm}$, as are shown in Fig. 2(a). Lines with dimensions of $50 \mathrm{~nm}$ were also produced and are shown in Fig. 2(b). For the analysis of the morphologies of structures with lateral dimensions of less than $40 \mathrm{~nm}$ reflection electron microscopy (REM), because of the high contrast and resolution, ${ }^{8}$ was used. In this technique, a dark-field image of the surface is obtained by tilting the electron beam into a bulk specimen until the specimen surface is almost parallel to the electron beam. A REM

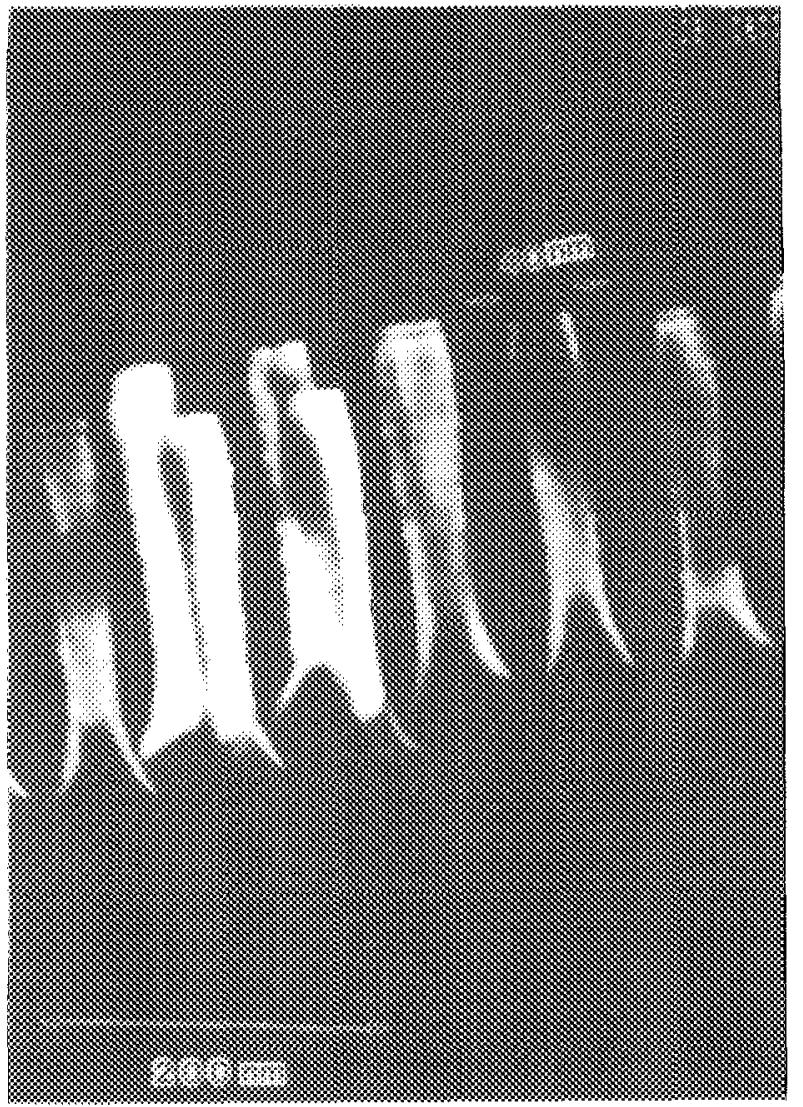

FIG. 3. REM image of columns etched into multiple quantum well material.

micrograph of etched columns is shown in Fig. 3. The dimensions of the structures can be determined accurately from this image using the etch depth, which is determined from depth profiles, as a standard. Lateral column dimensions ranging from 30 to $40 \mathrm{~nm}$ were measured in this way.

Contrast from individual quantum wells is obtained in the REM image, and a small amount of undercutting of the $\mathrm{Al}_{0.3} \mathrm{Ga}_{0.7}$ As layers can be seen in the form of steps on the sidewalls. This observation agrees well with reactive ion etch rate predictions for this gas mixture, according to which $\mathrm{Al}_{0.3} \mathrm{Ga}_{0.7} \mathrm{As}$ etches slightly faster than $\mathrm{GaAs}$ under low power and $\mathrm{BCl}_{3}$ concentrations used. ${ }^{7}$ However, contrast from the $\mathrm{GaAs} / \mathrm{Al}_{0.3} \mathrm{Ga}_{0.7}$ As superlattice is clearly visible throughout the column, indicating little disturbance of the sidewalls by the reactive ion etch. Reflection electron diffraction patterns, taken in the TEM, show bright Kikuchi line patterns on the etched surface. This is a further indication that only little damage of the material occurs in the top $10 \mathrm{~nm}$ of the sample.

Multiple quantum well structures have been patterned by electron beam lithography techniques to lateral dimensions of less than $50 \mathrm{~nm}$, the smallest to date. A liftoff and reactive ion etching technique has been developed to reproducibly make such structures in $\mathrm{GaAs} / \mathrm{Al}_{0.3} \mathrm{Ga}_{0.7}$ As layers. This is accomplished by using novel etch masks and etch gas mixtures at conditions providing only minor differences in the GaAs and AlGaAs etch rates.

The measurement of the dimensions of the produced 
structures, although still possible with standard SEM imaging, can be accomplished accurately by reflection electron microscopy. This technique also provides an understanding of the quality of the quantum wells, and is, therefore, very useful for analysis of the multiple quantum well structures.

The authors received part of their support from the Department of the Army, contract number DAAK20-85-C0395. The help of E. Beebe and L. Schiavone is also gratefully acknowledged. We thank M. Tamargo for the molecular beam epitaxially grown quantum well material.
'H. Sakaki, Jpn. J. Appl. Phys. 19, L735 (1980).

${ }^{2}$ M. A. Reed, R. T. Bate, K. Bradshaw, W. M. Duncan, W. R. Frensley, J.

W. Lee, and H. D. Shaw, J. Vac. Sci. Technol. B 4, 358 (1986).

${ }^{3}$ A. N. Broers, J. Electrochem. Soc. 128, 166 (1981).

${ }^{4}$ H. G. Craighead, J. Appl. Phys. 55, 4430 (1984).

${ }^{5}$ K. Kash, A. Scherer, J. Worlok, H. G. Craighead, and M. Tamargo, Appl. Phys. Lett. 49, 1043 (1986).

${ }^{6}$ M. B. Stern, H. G. Craighead, P. F. Liao, and P. M. Mankiewich, Appl. Phys. Lett. 45, 410 (1984)

${ }^{7}$ A. Scherer and H. G. Craighead (unpublished).

${ }^{8} \mathrm{H}$. G. Craighead, in The Physics and Fabrication of Microstructures and Microdevices, edited by M. J. Kelly (Springer, Berlin, 1986), p. 150. 